Documentation et bibliothèques

\title{
Recommandations pour le traitement des commandes de livres courants destinés aux bibliothèques
}

\section{Bookdealer — Library Relations Committee}

Volume 21, numéro 3, septembre 1975

URI : https://id.erudit.org/iderudit/1055393ar

DOI : https://doi.org/10.7202/1055393ar

Aller au sommaire du numéro

Éditeur(s)

Association pour l'avancement des sciences et des techniques de la

documentation (ASTED)

ISSN

0315-2340 (imprimé)

2291-8949 (numérique)

Découvrir la revue

Citer cet article

Bookdealer — Library Relations Committee (1975). Recommandations pour le traitement des commandes de livres courants destinés aux bibliothèques.

Documentation et bibliothèques, 21(3), 131-141.

https://doi.org/10.7202/1055393ar

Tous droits réservés (C) Association pour l'avancement des sciences et des techniques de la documentation (ASTED), 1975
Ce document est protégé par la loi sur le droit d'auteur. L'utilisation des services d’Érudit (y compris la reproduction) est assujettie à sa politique d'utilisation que vous pouvez consulter en ligne.

https://apropos.erudit.org/fr/usagers/politique-dutilisation/ 


\title{
Recommandations pour le traitement des commandes de livres courants destinés aux bibliothèques*
}

\author{
Préparées par le Bookdealer - Library Relations Committee**, Resources Section, \\ Resources and Technical Services Division, American Library Association \\ Traduites par Michel Thériault pour l'ASTED, avec l'autorisation du \\ détenteur du droit d'auteur, l'American Library Association
}

Le Bookdealer-Library Relations Committee, Resources Sections, Resources and Technical Services Division, American Library Association a préparé ces recommandations afin de conseiller les bibliothécaires et les fournisseurs. Le but de ce document est d'énoncer des recommandations sur les rapports entre bibliothécaires et fournisseurs dans le traitement des commandes de livres courants destinés aux bibliothèques. Nous incluons, dans le cadre de ces recommandations, les livres et brochures tels que définis dans le lexique de Library Statistics: a Handbook of Concepts, Definitions and Terminology (Chicago, American Library Association, 1966):

Livre: unité bibliographique indépendante ou volume d'une publication en série publié sous un titre uniforme, constituée de feuillets, feuilles, ou cahiers cousus ou reliés ensemble, avec ou sans couverture. On ne considère pas comme livres les volumes reliés de périodiques et de journaux. (N.B. Dans les présentes recommandations, ces volumes reliés de périodiques et de journaux peuvent être compris dans le processus de commande que nous

* Guidelines for Handling Library Orders for In-print Monographic Publications; prepared by the Bookdealer - Library Relations Committee of the Resources Section, Resources and Technical Services Division of the American Library Association. Chicago, American Library Association, 1974

* Bookdealer - Relations Committee: Abigail DahlHansen (jusqu'en 1971), Philip R. Dankert (jusqu'en 1971), W. Stuart Debenham, jr., Ashby J. Fristoe (jusqu'en 1971), M. Ann Heidbreder, John M. Bruer, Edna Laughrey, Murray S. Martin (président), Nancy Parker, Harriet K. Rebuldela (à partir de 1972), Leo R. Rift (représentant la Serials Section). Le comité remercie les bibliothécaires et les fournisseurs qui, par leurs suggestions, ont permis d'améliorer ces recommandations, spécialement Norman Dudley, Roma S. Gregory et Ronald Hagler de l'attention particulière apportée à la révision de ce texte. décrivons, en autant qu'ils sont commandés de la même façon que les livres, i.e. individuellement sans abonnement).

Brochure: unité bibliographique composée de quelques feuilles imprimées attachées ensemble de quelque façon, mais non reliées.

Les volumes publiés en collections font aussi l'objet des présentes normes. Toutefois, les publications en série et les périodiques en sont exclus. Nous nous attachons principalement aux éditeurs et fournisseurs américains. Ces normes ont été étudiées lors de réunions du Bookdealer-Library Relations Committee et de celles de la Resources Section. Elles ont été largement diffusées parmi les bibliothécaires, les libraires et les éditeurs.

\section{Rapports bibliothécaires-libraires}

Les bibliothécaires et les libraires entretiennent des rapports de nature contractuelle, qu'ils soient officialisés ou non. Ces relations ont pour objectif de procurer à la bibliothèque le meilleur service possible à un coût raisonnable.

Les présentes normes étudient plus particulièrement les aspects "service" du contrat. L'efficacité du service d'un fournisseur se reflétera sans doute dans ses prix, mais les coûts cachés supportés par la bibliothèque à cause de mauvais services sont parfois plus élevés que les économies apparentes réalisées en utilisant des services offerts à un coût moindre.

Les bibliothécaires doivent être conscients de l'importance de leur participation à l'amélioration de l'efficacité des services d'un fournisseur. Les responsabilités et les méthodes de travail de la bibliothèque doivent être constamment réévaluées pour assurer le meilleur rendement possible. Un manuel de procédures constamment tenu à jour nous apparaît 
essentiel. II n'est pas du ressort des présentes normes de traiter du choix des livres et du choix des fournisseurs; mais un choix judicieux effectué par des professionnels compétents va contribuer à rendre le système encore plus efficace et à en justifier les coûts.

Les rapports entre les parties seront couronnés de succès dans la mesure où toutes deux auront fait preuve de bonne volonté et de collaboration. Les bibliothécaires et les fournisseurs doivent se rencontrer fréquemment pour discuter de leurs difficultés, de leurs exigences et de leurs objectifs respectifs.

\section{Recommandations à l'intention des bibliothèques}

\section{Commandes de bibliothèque}

\section{I-Bons de commande}

C'est à la bibliothèque que revient la responsabilité de concevoir et de faire imprimer les bons de commande. Une seule formule normalisée ne semble pas une solution adéquate vu les exigences particulières de chaque bibliothèque ${ }^{1}$. Néanmoins, certains renseignements sont essentiels et leur localisation sur les bons de commande devrait être toujours la même. Les formules devraient répondre à la fois aux exigences du bibliothécaire et du fournisseur. Les bibliothèques devraient donc consulter leurs principaux fournisseurs avant d'en préparer de nouvelles.

\section{A. Conception et disposition}

On utilise généralement deux types de bons de commande: des bons individuels (pour chaque titre commandé) ou des listes. Les deux sont acceptables et supposent un modèle différent mais simple et intelligible. Dans les deux cas, il faut identifier chaque formule comme un bon de commande et y inscrire le nom de la bibliothèque et celui du fournisseur.

\section{Bons de commande individuels}

a) Utiliser un bon de commande individuel pour chaque titre.

b) Indiquer clairement chaque élément; ex. "auteur", "titre», "quantité", etc. Les données non identifiées, spécialement les chiffres, sont déroutantes.

c) Grouper les éléments de façon à sépa-

1. Note du traducteur: Un sous-comité du comité Z.39 de l'American Standards Association travaille actuellement sur une formule de bon de commande normalisée. rer, autant que possible, les renseignements à l'usage de la bibliothèque et ceux destinés aux fournisseurs. Cette distinction est très importante, sans quoi on risque d'occasionner des erreurs de part et d'autre.

d) Prévoir l'espace suffisant aux endroits destinés à recevoir des informations de longueur variable: ex. auteur, titre, collection.

e) Ne pas juxtaposer des éléments qui se ressemblent; ex. la numérotation internationale normalisée (ISBN) et le numéro de la fiche de la Library of Congress.

f) Spécifier la fonction de chacun des feuillets du bon de commande multiple. Si un feuillet doit être retourné avec le livre, l'indiquer sur ce feuillet.

\section{Listes}

a) Préparer une liste par éditeur; du moins, regrouper les titres par éditeur sur une même liste.

b) Assigner un numéro de commande à chacune des listes et identifier chaque titre. Utiliser de préférence une combinaison alpha-numérique, ex. commande no 99 , titre $A, B, C$, etc.

Sur chaque commande, il faut indiquer clairement où expédier les factures, la correspondance et la marchandise commandée. II est aussi souhaitable de laisser l'espace nécessaire au fournisseur afin qu'il y note ses propres remarques. Si la bibliothèque annule les commandes en suspens après un délai déterminé, elle doit le préciser sur le bon de commande (Voir aussi IV - Annulations).

N'inscrire que les éléments propres au processus de commande. Des notes à l'usage de la bibliothèque telles que "mettre en réserve pour le cours BIBL. 610" ou "pour la bibliothèque de droit" compliquent le travail du fournisseur. Éviter l'emploi d'abréviations qui peuvent porter à confusion "Ǵt. Brit.", ou "U.S." sont des vedettes de collectivité-auteur acceptables dans un système manuel de classement, mais peuvent occasionner des erreurs d'identification et de classement dans un système automatisé. Inscrire au complet, dans le titre, des phrases telles que «Introduction à" ou "Histoire abrégée de»; ceci facilite l'identification des titres. Surtout, éviter l'emploi d'initiales équivoques, ex. "O.U.P.» qui peut signifier Oxford, Oregon ou Oklahoma University Press. 
B. Données essentielles

\section{Elements identifiant le titre commandé}

a) Auteur. Etre aussi complet que pratique. Dans les notices ayant un auteur personnel, mettre d'abord le patronyme. Soigner les vedettes de collectivité-auteur. On ne devrait pas entrer un ouvrage de l'Air Environment Center de la Pennsylvania State University sous la seule vedette "Pennsylvania State University"; dans les systèmes automatisés ou mécanisés, ne pas couper la vedette après un nombre pré-établi de caractères.

b) Titre. Inscrire intégralement le titre, y ajouter le sous-titre s'il y a lieu, surtout si le titre est général: ex. Sir George Grey: Pioneer of Empire.

c) Support. Préciser quelle présentation de l'ouvrage vous commandez, ex. broché, relié, éd. de poche, ainsi que vos exigences particulières, ex. "Library ed.». "Text ed.», «Ed. anglaise».

d) Editeur. Fournir le nom de l'éditeur au complet ainsi que les nom et adresse de la société ou de l'établissement; si vous ne les connaissez pas, il est bon de le mentionner: ex. la Pennsylvania State University possède au moins vingt éditeurs (différents départements ou centres de recherche) qui ont chacun leur adresse spécifique; ne pas préciser le nom ou l'adresse peut signifier un retard de plusieurs semaines dans la livraison de la commande.

e) Lieu. Indiquer le nom de la ville et s'il y a lieu de distinguer entre des villes de même nom, ajouter l'état, la province ou le pays, ex. London (Ont.), London (Engl.).

f) Date. Préciser la date de publication de l'édition commandée, celle du copyright étant la meilleure.

g) Éditeur intellectuel. Inscrire le nom de l'éditeur intellectuel s'il n'apparaît pas dans la notice principale.

h) Edition. Préciser le numéro ou un autre élément décrivant l'édition: ex. $2 e$ éd., $2 e$ éd. rév. Être explicite.

i) Collection. Ajouter le nom de la collection si cela peut aider à l'identification d'un ouvrage; inclure le numéro de collection du titre demandé: ex. California Publications in Classics, no. 1.

j) Prix. Donner le prix de catalogue en s'assurant qu'il s'agit bien de celui de l'édition commandée; par exemple, ne pas inscrire le prix de l'édition de poche si vous voulez l'édition reliée.

k) Numérotation internationale normalisée du livre (ISBN). Préciser ce numéro s'il est connu. Même si on n'utilise pas encore universellement ce système, il permet une identification précise de l'éditeur, du titre, de l'édition. Ne pas confondre avec les numéros d'inventaire donnés aux éditions reliées ou de poche d'un même titre («stock number").

I) Nombre de volumes. Indiquer le nombre de volumes de l'ouvrage si possible. Si vous ne commandez qu'un seul tome d'un ouvrage, le spécifier, ex. vol. 2 seulement (Voir $2 b$ ).

\section{2. Éléments propres au processus de com- mande}

Ces éléments concernent l'opération commande-vente-achat et les activités qui l'entourent plutôt que le titre demandé. On verra à les grouper sur le bon de commande.

a) Numéro et date de la commande.

b) Nombre d'exemplaires commandés. Le préciser, ex. un exemplaire de chaque volunie, ou un exemplaire des volumes 1 à 6 seulement, ou encore 6 exemplaires.

c) Commandes urgentes. Le noter clairement et, tel qu'indiqué plus bas, n'utiliser que lorsque ceci s'avère nécessaire.

d) Instructions particulières. Elles ne s'appliquent qu'à un titre particulier; ex. préciser le prix avant d'expédier; facturer séparément.

e) Instructions générales. Elles valent pour l'ensemble des commandes et comprennent l'adresse de livraison des livres, celle pour l'expédition de la facture et de la correspondance; elles devraient être imprimées sur le bon de commande; faire un renvoi au recto, si elles apparaissent au verso du bon de commande. Éviter les noms de per- 
sonnes dans les adresses de livraison, sauf si l'établissement l'exige.

Si le nombre de transactions avec un fournisseur le justifie, il peut s'avérer économique de faire imprimer des bons de commande à son nom et d'y inscrire d'autres détails particuliers aux commandes placées chez lui.

\section{II - Envoi des bons de commande}

Expédier les commandes régulièrement; ne pas accumuler indéfiniment des commandes ordinaires pour ensuite les expédier en bloc, ce qui surcharge le fournisseur et retarde l'exécution de l'ensemble des commandes de la bibliothèque. Poster les commandes au moins une fois la semaine, même s'il y en a peu.

Lorsque des commandes doivent être expédiées en bloc, par exemple pour des volumes en réserve, des collections à mettre dans des classes ou des collections d'usuels, vous entendre au préalable avec le fournisseur. Ces commandes doivent être envoyées longtemps d'avance avec une lettre stipulant les délais de livraison. La plupart du temps, le fournisseur demandera 6 à 8 semaines pour la livraison, compte tenu de la localisation des maisons d'édition où il place ses commandes et de son propre stock.

Développer des méthodes appropriées aux commandes urgentes ou spéciales. Définir le sens du terme urgent d'après vos exigences et ne pas en abuser, expliquer à vos fournisseurs ce que vous entendez par urgent et demander leur collaboration. Etre prêt à assumer les frais de services spéciaux requis du fournisseur (courrier par avion ou livraison express).

\section{III - Relances}

A. Avant de relancer les commandes d'ouvrages en suspens, les bibliothécaires devraient:

1. Essayer de déterminer si l'ouvrage est publié ou non; dans ce dernier cas, ne pas relancer la commande.

2. Conserver une mention des rapports du fournisseur: ne pas rappeler une commande quand, une semaine auparavant, celui-ci vous a répondu "pas en magasin - commandons".

3. Laisser un intervalle suffisant entre la commande et la relance. Les bibliothécaires peuvent normalement s'attendre à recevoir les ouvrages dans les limites de temps indiquées plus bas et devraient donc élaborer leur système de rappel en conséquence:

\section{a) Éditeurs commerciaux des États-Unis.}

Délais de livraison: entre 30 et 60 jours; après 45 jours, la bibliothèque devrait avoir obtenu une réponse - soit la livraison ou un rapport - sur au moins $60 \%$ des titres commandés.

N.B. Les volumes disponibles sur le marché, mais publiés il y a au moins trois ans exigent plus de temps. Prévoir 90 jours pour la livraison de ces titres, mais $50 \%$ de ces commandes devrait avoir été fourni en deçà de 60 jours.

b) Éditeurs "non commerciaux» des Etats-Unis.

Les titres récents devraient avoir été fournis avant 90 jours; pour les ouvrages moins connus, attendre 120 jours.

N.B. Les bibliothécaires peuvent accélérer la livraison en indiquant au fournisseur l'adresse complète de l'éditeur ou en commandant directement.

c) Éditeurs "commerciaux" étrangers

La plupart des ouvrages en provenance d'Europe occidentale peuvent être fournis en deçà de 150 jours. La livraison des ouvrages édités dans d'autres pays exigera souvent plus de temps.

N.B. Les bibliothécaires doivent s'assurer que leurs fournisseurs soient capables et désireux de traiter les commandes des publications non commerciales ou étrangères; dans plusieurs cas et c'est ce que conseillera un bon fournisseur - il est préférable de se les procurer directement; quant aux publications étrangères, il est souhaitable d'avoir un fournisseur dans le pays d'origine ou de faire appel à un fournisseur spécialisé.

En choisissant un mode de commande pour ce genre d'ouvrages, bibliothécaires et fournisseurs doivent établir clairement leurs attentes et leurs possibilités respectives. Chacun y perd quand un fournisseur offre ses services pour des commandes qu'il ne peut traiter 
efficacement. Réciproquement, les bibliothécaires doivent être réalistes quant à ce type de service et quant à son coût. Voilà donc les délais auxquels les bibliothécaires devraient s'attendre. Ils doivent tenir compte cependant de certaines variations: par exemple, le titre d'un document peut avoir été modifié entre le moment de l'émission de la commande et celui de sa livraison; s'il n'y a eu aucun rapport du fournisseur sur ces commandes en cours, les bibliothécaires devraient alors pouvoir les annuler.

d) Espacer suffisamment les relances pour permettre au fournisseur de communiquer avec l'éditeur et de vous répondre. Dans la plupart des cas, prévoir au moins quatre semaines entre chaque rappel fait aux États-Unis. Le courrier par avion peut être utilisé, mais il augmente le coût final de votre commande. Émettre un second rappel après 30 jours si nécessaire.

B. Identifier clairement toutes les relances et $y$ inscrire tous les renseignements nécessaires pour chacun des titres.

1. On peut se servir de lettres ou de formules, mais il est préférable d'utiliser une copie du bon de commande original en mettant bien en évidence qu'il s'agit d'une relance; libellé suggéré: Première relance (date) Ceci n'est pas une commande.

2. La relance doit comporter l'espace suffisant pour la réponse du fournisseur et l'indication de la retourner dûment remplie.

3. Si vous n'utilisez pas une copie du bon de commande, assurez-vous que le fournisseur reçoit tous les détails relatifs au titre réclamé, ex. numéro de commande, tome du volume relancé d'un ouvrage en plusieurs volumes, ou quantité précise réclamée sur le nombre d'exemplaires commandés.

La meilleure réponse à une relance est évidemment de recevoir l'ouvrage. Cependant, s'il faut faire une ou deux relances avant de recevoir une bonne partie des commandes, le bibliothécaire doit faire enquête. Si les méthodes en usage à la bibliothèque sont déficientes, les corriger et en discuter avec le fournisseur. Si le service demeure insatisfaisant, l'unique recours sera de changer le fournisseur.

\section{IV - Annulations}

De concert avec vos principaux fournisseurs, mettre au point un mode d'annulation de commandes. Annuler les commandes en suspens après un délai fixe (cf. III, A, 3), à moins qu'entre-temps, on vous ait fourni des renseignements indiquant que la commande est en cours de traitement. Cette mesure permet d'examiner périodiquement le dossier des commandes et d'éviter d'engager une trop grande proportion des crédits et décourage la pratique arbitraire d'annuler et de recommander ailleurs. Dans les délais convenus, un fournisseur a le droit de s'attendre à ce qu'une bibliothèque achète effectivement les ouvrages qu'elle a commandés.

Les politiques budgétaires d'un établissement exigent parfois que les budgets soient dépensés à une date précise. La bibliothèque devra donc annuler toutes les commandes non livrées avant de libérer les crédits engagés et de les dépenser ailleurs.

Les commandes en double faites par erreur peuvent être annulées; on conviendra alors des arrangements nécessaires avec le fournisseur. Le retour d'un ouvrage au fournisseur est onéreux; celui-ci acceptera à l'occasion une perte pour rendre service à un client; une telle pratique doit demeurer exceptionnelle.

Les bibliothécaires devraient établir le coût total d'un retour d'ouvrage (prix de l'ouvrage plus frais d'administration) et ainsi établir le montant en deçà duquel un ouvrage ne sera pas retourné. (Les frais d'administration des retours varient d'une bibliothèque à l'autre, mais peuvent facilement dépasser $\$ 3.00$ en plus du prix de catalogue). S'il y a un taux élevé de commandes en double, il y a probablement des failles au niveau de la recherche bibliographique et du processus de commande.

\section{V - Retours}

En consultation avec les fournisseurs concernés, décider des règles à suivre pour retourner des ouvrages et les inscrire dans votre manuel de procédures.

Inclure, avec chaque retour, les notes explicatives nécessaires et indiquer la date. On peut utiliser une lettre polycopiée sur laquelle on cochera la raison du retour; ex.:

mauvais titre mauvaise édition envoi en double trop d'exemplaires fournis 
non commandé

exemplaire défectueux

Dans chaque lettre, référer à votre numéro de commande et au numéro de facture du fournisseur et demander les corrections nécessaires: ex. "nous suspendons le paiement de la facture jusqu'à la livraison du bon titre", ou "veuillez nous faire parvenir une note de crédit».

\section{A. Mauvais titres}

1. Vous pouvez retourner automatiquement un titre non commandé. Si la bibliothèque est responsable de l'erreur, il faut demander une autorisation de retour.

2. Retourner l'ouvrage non estampillé. Prévoir l'estampillage des nouveautés après les opérations de réception et de vérification.

\section{B. Ouvrages défectueux}

1. Retourner les ouvrages défectueux dès leur réception pour les remplacer. Si on découvre des défectuosités après utilisation de l'ouvrage, il importe de ciistinguer entre les défauts de fabrication et l'usure normale.

2. S'entendre avec le fournisseur sur la façon d'effectuer le retour de l'ouvrage: certains éditeurs acceptent le retour de la page de titre seulement, avec une note expliquant les défauts; ceci s'avère la méthode la plus économique.

3. Spécifier les défectuosités de l'ouvrage dans la lettre qui accompagne le retour.

\section{Correction de la facture}

1. Se soumettre à la fois aux exigences comptables du fournisseur et de la bibliothèque. (Voir VI, B, 5).

\section{VI - Exigences d'ordre financier}

Simplifier le plus possible les exigences de facturation et de paiement. Certains états ou gouvernements locaux (comtés, municipalités) ont des exigences de nature légale, mais la plupart des règlements viennent des établissements eux-mêmes.

Les bibliothécaires doivent se familiariser avec les méthodes comptables de leur établis- sement. De plus, il est essentiel que les bibliothèques fassent prendre conscience aux services administratifs concernés que l'acquisition de livres est un processus constant et courant qui ne se prête pas aux contrôles-surprise, ni au traitement par lots, ni, sauf dans certains cas, à l'établissement d'échéanciers dans les opérations. Dans le cas de certains titres très coûteux, éviter d'exiger une confirmation de la commande ou du prix. C'est un procédé aride et une perte de temps de transcrire les données des factures ou d'autres formules sur des bordereaux internes normalisés.

Plusieurs établissements possèdent un système comptable automatisé; ces systèmes prévoient des périodes fixes pour le règlement des factures et d'autres périodes où ce système n'est pas en opération, surtout à la fin de l'exercice financier. II peut y avoir, chaque mois, des périodes prévues pour la vérification et le paiement de certaines catégories de factures. Bien connaître les méthodes comptables et aussi le traitement appliqué aux cas d'exception: en informer vos fournisseurs. Si certaines de ces méthodes vous causent préjudice, en discuter avec les services administratifs de votre établissement et en établir de plus efficaces.

\section{A. Modes de facturation}

Bien que les systèmes comptables varient beaucoup d'un établissement à l'autre, quelques recommandations générales sur les modes de facturation s'imposent.

1. Informer clairement les fournisseurs du mode de facturation requis, c'est-à-dire une facturation séparée pour chaque commande ou une facturation groupant plusieurs numéros de commande. Règle générale, ne demander une facturation séparée que pour les commandes engagées sur des fonds spéciaux qui amènent des modes particuliers de paiement. De telles commandes devraient être clairement libellées comme suit et en caractères gras: «FONDS SPÉCIAL - S.V.P. FACTURER SÉPARÉMENT». Éviter d'employer une méthode qui obligerait le fournisseur à se reporter à d'autres dossiers.

2. Avertir les fournisseurs si votre système comptable mécanisé ne permet pas de payer plus d'un certain nombre de factures avec un même chèque permettant ainsi au fournisseur de maintenir à jour ses comptes-clients et d'éviter l'envoi inutile de demandes de paiement. 
3. Informer vos fournisseurs et demander leur collaboration, s'ils doivent traiter séparément les commandes de livres, les abonnements aux périodiques et les commandes de publications en série.

4. Vérifier régulièrement les adresses des services où doivent être acheminées les factures. Retenir la fonction plutôt que le nom des personnes, ex. "Chef du service des acquisitions». Avertir rapidement les fournisseurs des changements.

5. N'exiger que les copies de facture nécessaires au traitement de la commande. Trois exemplaires devraient normalement suffire (i.e. un original et deux autres copies).

\section{B. Procédures de paiement}

1. Acquitter rapidement afin de vous assurer d'un bon service. La plupart des factures devraient être réglées dans les trente jours. Éviter des délais de 90 jours ou plus qui entraînent un coût supplémentaire.

2. Informer le fournisseur des contraintes de temps qui échappent au contrôle de la bibliothèque et éviter que vos propres méthodes ne soient cause de retard.

3. Ne grouper les factures que si vous avez pris entente avec le fournisseur pour payer à toutes les deux semaines ou à tous les mois ou encore si la bibliothèque reçoit un nombre de factures tel qu'il s'avère économique de les retenir pour les régler à intervalles fixes, par exemple à tous les dix jours.

4. Retenir, au plus 30 jours, les factures d'envois incomplets. Tout en tenant compte que les délais de livraison peuvent s'échelonner sur deux ou trois semaines, relancer les titres en suspens après 30 jours et faire un paiement partiel.

5. Élaborer des règles pour le traitement des envois incomplets, des retours et des erreurs; il existe maints usages: vous entendre avec le fournisseur sur celui qui vous convient le mieux:

a) La note de crédit. La facture sera habituellement retenue jusqu'à la réception de la note de crédit.

b) En rayant des titres sur la facture. Avertir le fournisseur des titres corrigés en lui envoyant copie de sa facture révisée.

c) En demandant une facture corrigée. C'est sans doute la méthode la plus lente, mais la plus convenable quand la majeure partie de la facture demande correction ou si les modifications sont complexes.

d) En retournant la facture avec la marchandise si aucun autre titre n'y apparaît.

6. Se rappeler de noter sur le bon de commande ou ailleurs les démarches entreprises au sujet des notes de crédit ou des paiements partiels.

7. Indiquer le numéro de facture sur le chèque ou donner la liste des numéros de factures payées avec chaque règlement.

8. Se conformer aux exigences particulières des fournisseurs ou éditeurs, ex. "retourner la carte perforée avec le chèque»; si cela faisait problème à l'établissement, en discuter avec le fournisseur.

9. Être prêt à vérifier les états de compte des fournisseurs, que cela se fasse à la bibliothèque ou au service de la comptabilité de l'établissement. Les numéros de facture et les sommes facturées devraient suffire à vérifier leur exactitude.

\section{Recommandations à l'intention du fournisseur}

\section{I-Formules accompagnant les ouvrages non requis par un bon de commande distinct.}

Quand, suite à un accord préalable, les fournisseurs expédient des livres aux bibliothèques (ex. ouvrages en plusieurs volumes, collections, titres envoyés pour examen, ou à la suite d'une commande globale) les bibliothécaires devraient recevoir tous les détails pertinents aux titres fournis. Ceci réduit le temps consacré au traitement et simplifie la manipulation des titres envoyés pour examen.

Imaginer des formules qui donneront tous les renseignements nécessaires. Ce peut être sous forme de listes ou une combinaison commandes / factures individuelles. Dans chaque cas, indiquer dans la notice de' chaque titre les éléments principaux suggérés plus haut pour la commande (I, B, 1). Le livre étant fourni en même temps que cette formule, ces éléments peuvent être simplifiés dans leur présentation, mais il faut éviter les codes ou les notices trop abrégés. 
Les bibliothécaires savent que les règles de catalogage des vedettes de collectivitésauteur ou d'éditeurs intellectuels sont complexes. Les erreurs dans ces vedettes sont compréhensibles, bien que non souhaitables; cependant, rédiger une notice au nom de l'éditeur intellectuel plutôt qu'à celui de l'auteur de l'ouvrage cause des problèmes.

Dans toute correspondance comme sur toute formule, référer au numéro du bon de commande de la bibliothèque, à une lettre ou encore à un code indiquant la raison de l'envoi, ex. commande globale no 66/2, ou commande globale (lettre du 01.07.70).

\section{II - Exécution de la commande}

\section{A. Période d'attente}

Les bibliothécaires peuvent raisonnablement s'attendre à recevoir leurs commandes dans les délais suivants:

1. Éditeurs commerciaux des États-Unis. Livraison des commandes: entre 30 et 60 jours; après 45 jours, vous devriez avoir donné réponse sur au moins $60 \%$ des titres commandés (soit la livraison, soit un rapport).

N.B. Les volumes disponibles sur le marché, mais publiés il y a au moins trois ans exigent plus de temps. Prévoir 90 jours pour la livraison de ces titres, mais $50 \%$ de ces commandes devrait avoir été fourni en deçà de 60 jours.

2. Éditeurs "non commerciaux" des ÉtatsUnis.

Les titres récents devraient avoir été fournis avant 90 jours; pour les ouvrages moins connus, attendre 120 jours.

3. Éditeurs "commerciaux» étrangers. La plupart des ouvrages en provenance d'Europe occidentale peuvent être fournis avant 150 jours. La livraison des ouvrages édités dans d'autres pays exigera souvent plus de temps.

\section{B. Modes d'approvisionnement}

1. Ne pas grouper les envois si cela crée un délai de plus de deux jours, à moins qu'il n'y ait eu un accord préalable qui l'autorise.

2. Livrer immédiatement les commandes URGENTES. Facturer à la bibliothèque les frais supplémentaires encourus. Bibliothécaire et fournisseur devraient s'entendre sur le mode de transport à utiliser.

\section{III - Rapports sur les commandes en sus- pens}

\section{A. Généralités}

1. Que les rapports sur l'état des commandes soient clairs et non ambigus.

2. Transmettre aux bibliothèques un rapport sur toutes les commandes en suspens avant la fin de la période d'attente mentionnée ci-haut (II).

3. Sur chaque rapport, fournir les renseignements suivants:

a) Numéro de commande de la bibliothèque (et numéro du titre s'il s'agit d'une liste) et date.

b) Description bibliographique sommaire, suffisante pour identifier l'ouvrage.

c) Le contenu du rapport lui-même et le détail des mesures prises.

d) Date du rapport.

4. Si les rapports sont rédigés sur la formule de relance de la bibliothèque, les éléments mentionnés ci-haut (no 3 ) peuvent être modifiés en conséquence.

5. Fournisseur et bibliothécaire doivent garder un dossier des rapports et relances.

N.B. Les systèmes automatisés ou mécanisés exigent de l'exactitude dans l'emploi des lettres et des chiffres utilisés pour assortir les différents dossiers. Par conséquent, s'assurer de transposer correctement lettres et chiffres.

\section{B. Rapports suggérés}

1. Rapports reliés aux activités du fournisseur

a) Pas encore reçu de l'éditeur (Not yet received from the publisher - NYR). Ceci doit vouloir dire que le fournisseur a déjà fait la commande auprès de l'éditeur ou de l'agent et qu'il a déjà fait rappel.

b) Pas en magasin - Commandons (Out of stock - Ordering). Ceci doit vouloir dire que le fournisseur lui-même n'a plus d'exemplaires dans son entrepôt et que des exemplaires supplémentaires du titre ont été commandés chez 
l'éditeur. Si possible, fournir une date approximative de livraison.

c) Relançons (Claiming). Ceci doit vouloir dire que le fournisseur suit sa commande de près et a déjà relancé.

\section{Rapports reliés aux activités de l'éditeur}

a) Pas encore publié (Not yet published - NYP). Ceci fait suite aux renseignements reçus de l'éditeur et vérifiés dans les sources disponibles. Le rapport devrait aussi donner, si possible, une date approximative de publication.

b) Pas en magasin chez l'éditeur (Out of stock - Publisher), Réimpression en cours (Reprinting) ou $\dot{A}$ la reliure (Binding). Ces différents renseignements font partie du même rapport, font référence aux déclarations de l'éditeur lui-même et incluent une date approximative de livraison. Un rapport supplémentaire, "En commande» (On order), peut être utilisé si le fournisseur s'approvisionne chez un agent représentant un ou plusieurs éditeurs. Si la date de livraison n'est pas précisée, il est préférable d'annuler la commande.

c) Epuisé (Out of print - OP). Ces rapports sont généralement vrais. Mais quand certains éditeurs voient leur approvisionnement diminuer, ils préfèrent ne fournir que des commandes venant directement de clients individuels et non de fournisseurs de bibliothèque. Les fournisseurs devraient s'en assurer et en avertir les bibliothécaires, en envoyant le rapport «Épuisé - annulé", le meilleur dans les circonstances.

N.B. Bibliothécaire et fournisseur devraient se mettre d'accord sur une façon de traiter les commandes d'ouvrages épuisés. Si un fournisseur offre de rechercher ces titres, développer une méthode pour ce faire en spécifiant une date-limite. Généralement, la plupart des bibliothécaires et des fournisseurs préfèrent que ces commandes soient traitées indépendamment des commandes d'ouvrages disponibles. Si tel est le cas, les bibliothécaires devraient accepter le rapport "Épuisé" comme une annulation de la part du fournisseur.

\section{Rapports divers}

a) Pas $d u$ fonds (Not our publication NOP). C'est un rapport reçu par un fournisseur et venant d'un éditeur. Le fournisseur devrait vérifier de nouveau le catalogue de l'éditeur et examiner les divergences entre le catalogue et le rapport. Si le fournisseur transmet une réponse "Pas du fonds" à un bibliothécaire, la commande est considérée comme annulée. Si le bibliothécaire veut commander l'ouvrage de nouveau après avoir trouvé des renseignements plus précis, cette demande sera traitée comme une nouvelle commande.

b) Mauvais titre fourni. Si l'éditeur ou l'agent expédie au fournisseur un mauvais titre, envoyer un rapport au bibliothécaire expliquant le délai.

c) Exemplaire défectueux. Même remarque que pour $b$.

d) Quantité insuffisante fournie, etc. Si une quantité insuffisante d'exemplaires est fournie, les expédier au bibliothécaire avec un rapport indiquant que les autres suivront plus tard.

\section{IV - Facturation}

Les factures seront libellées clairement et exactement. Expédier à la bibliothèque deux copies ou plus de l'original.

\section{A. Contenu de la facture}

1. Nom et adresse du fournisseur où le paiement doit être envoyé.

2. Numéro(s) de commande de la bibliothèque (et numéro(s) du (des) titre(s) s'il y a lieu).

3. Renseignements bibliographiques sur chaque titre. L'auteur et le titre sont un minimum. Utiliser la notice apparaissant sur le bon de commande.

N.B. Ne pas utiliser des abréviations codées ou des chiffres, etc. Cela cause des délais dans la réception et le paiement. Si le titre fait partie d'une collection, le nom de la collection et le numéro du titre ne suffisent pas.

4. Nombre d'exemplaires fournis. (Si ce nombre ne correspond pas au nombre commandé, en expliquer la différence).

5. Nombre de volumes fournis, en les identifiant, ex. vol. 1 à 6 , ou 2 v.

6. Prix de chaque titre: le prix de catalogue et le prix net payé par la bibliothèque. 
7. Les autres frais: service, taxes, poste, etc.

8. Quand l'énumération nécessite plus d'une page, indiquer clairement à chaque page le montant reporté et le montant total devant être payé. Ne pas indiquer le prix total seul sur une page séparée, puisqu'elle peut facilement se détacher de la liste détaillée et retarder la vérification et le paiement.

\section{B. Précautions à prendre}

1. N'inclure aucun rapport pour les titres non fournis sur la facture. Celle-ci ne devrait énumérer que les titres effectivement fournis.

2. Ne pas énumérer divers types de documents (livres, périodiques et suites) sur une même facture. Ces ouvrages sont traités de façon différente les uns des autres par les bibliothèques; les rassembler sur une même facture peut en retarder le règlement.

3. Ne pas énumérer trop de titres sur chaque facture. En fait, à chaque facture doit correspondre un envoi.

4. Facturer séparément les titres envoyés directement par un éditeur à une bibliothèque («drop shipments»). Si des factures énumèrent ces titres envoyés directement de l'éditeur et des titres envoyés normalement par le fournisseur, une erreur dans la livraison d'un titre peut retarder la vérification et le paiement de la facture.

5. Facturer séparément les titres qui sont à livrer à différentes adresses. Le traitement de ces commandes, à l'intérieur de l'établissement, se fait habituellement de façon différente suivant l'adresse de livraison.

6. S'assurer de facturer séparément quand on en fait la demande, plus particulièrement lorsqu'il s'agit de titres dont le paiement s'effectue à même des fonds spéciaux. Ce genre d'erreur peut parfois être réglé par le bibliothécaire, mais il faut souvent facturer à nouveau.

\section{Envoi des factures}

Envoyer les factures avec les ouvrages facturés. Quand il s'agit de livraison directe de la part de l'éditeur (mais facturée par le fournisseur) ou d'expédition en plusieurs colis, l'envoi des factures est fait indépendamment des ouvrages, mais il faut veiller à ce que l'arrivée des factures coïncide au mieux avec celle des colis.

Les colis sans facture devraient contenir un bordereau de livraison. Si ce bordereau est une copie de la facture, il devrait contenir une indication claire, ex. "Copie de facture" ou "Bordereau de livraison", afin d'éviter une confusion possible avec la facture originale et un paiement en double, le cas échéant. Indiquer clairement sur la facture lorsqu'elle est payable à l'avance.

\section{Etats de compte}

Les états de compte énumérant les factures encore impayées doivent se distinguer de façon précise des factures. En effet, trop de ces états de compte, surtout ceux produits mécaniquement, ressemblent à s'y méprendre à des factures et peuvent ainsi occasionner des paiements en double.

II faut se rappeler que les bibliothécaires ne sont habituellement pas des comptables et que l'objet de chaque formule doit être exprimé clairement.

\section{Recommandations générales}

L'objectif d'ensemble est d'associer le mieux possible service et coût. Des coûts trop réduits et un service excellent sont des concepts incompatibles; des coûts trop élevés et un mauvais service sont des concepts dont la juxtaposition est inacceptable. Les bibliothécaires peuvent et doivent s'attendre à faire les frais d'un bon service, mais ils sont, dès lors, en droit de l'obtenir.

\section{Bibliothécaires}

Choisissez avec soin vos fournisseurs. Assurez-vous qu'ils comprennent quels genres d'ouvrages ils auront à traiter. Certains genres d'ouvrages (venant de sociétés savantes et associations ou ouvrages à remise réduite) posent des problèmes particuliers aux fournisseurs; vous ne pouvez vous attendre à ce qu'ils les traitent aussi facilement et à aussi bon compte que les ouvrages dans le commerce. Assurez-vous aussi que vous vous entendez bien sur les remises, les frais de service et aussi sur les règles à suivre. Respectez les engagements que vous avez pris. Discutez avec le fournisseur de vos attentes et de vos problèmes et comprenez les siens.

\section{Fournisseurs}

Les bibliothécaires ont eux aussi leurs 
problèmes. Ne promettez pas au-delà de vos possibilités. Discutez ouvertement de vos problèmes avec les bibliothécaires. N'oubliez pas que les bibliothécaires contrôlent rarement la totalité du système au sein duquel ils travaillent, en particulier l'aspect financier. Ils consentent habituellement à étudier les possibilités de modifications éventuelles. Surtout, restez toujours en communication. Les bibliothécaires doivent pouvoir expliquer à leurs clients la raison des délais encourus et des autres problèmes qui peuvent surgir. II n'y a rien de mieux pour améliorer le service et venir en aide à la fois au bibliothécaire et au fournisseur qu'un échange complet de renseignements.

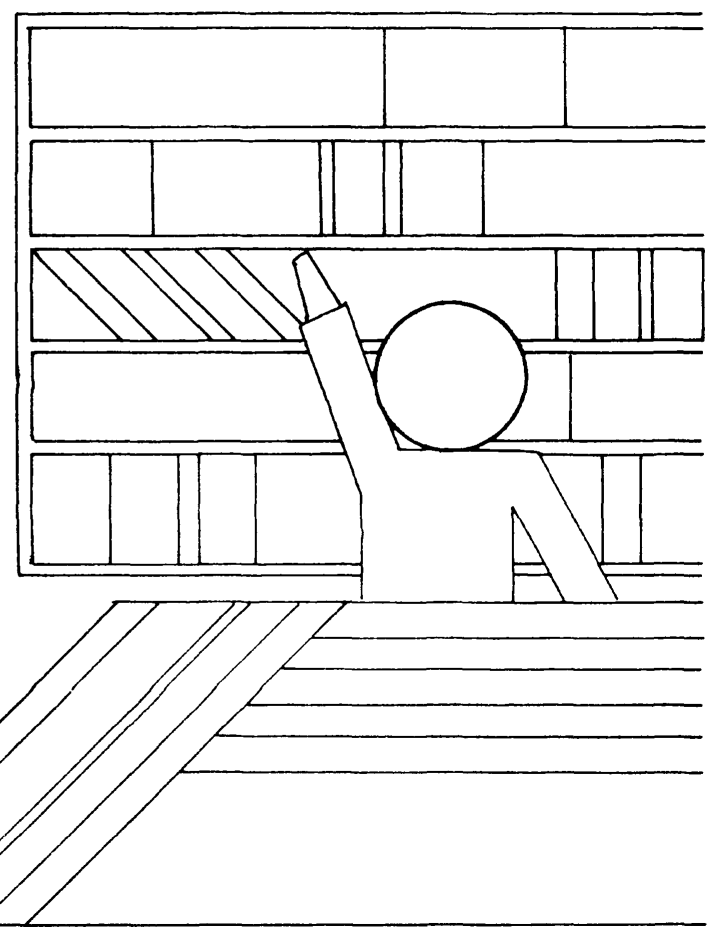

\section{À votre service}

\section{DES TITRES}

dans les disciplines suivantes:

Études médiévales, folklore, histoire, pastorale, philosophie, questions nationales, questions sociales, science politique, spiritualité, théologie.

\section{EDUCATION ET SOCIETE} revue d'animation

\section{RELATIONS}

revue d'intérêt général

L'abonnement: $\$ 8$

L'abonnement $\$ 5$

\section{SCIENCE ET ESPRIT}

revue philosophique et theologique

L'abonnement: $\$ 7$

\section{PHILOSOPHIQUES}

revue de philosophie

L'abonnement: \$8

\section{Les Éditions Bellarmin}

8100 , boul. Saint-Laurent 